\title{
A Vertical Handoff Decision Algorithm for Heterogeneous Wireless Networks
}

\author{
Enrique Stevens-Navarro, Vincent W.S. Wong, and Yuxia Lin \\ Department of Electrical and Computer Engineering \\ The University of British Columbia, Vancouver, Canada \\ E-mail: \{enriques, vincentw, yuxial\}@ece.ubc.ca
}

\begin{abstract}
One of the major design issues in heterogeneous wireless networks is the support of vertical handoff. Vertical handoff occurs when a mobile terminal switches from one network to another (e.g., from WLAN to CDMA 1xRTT). The objective of this paper is to determine the conditions under which vertical handoff should be performed. The problem is formulated as a Markov decision process. A link reward function and a signaling cost function are introduced to capture the tradeoff between the network resources utilized by the connection and the signaling and processing load incurred on the network. A stationary deterministic policy is obtained when the connection termination time is geometrically distributed. Numerical results show good performance of our proposed scheme over two other vertical handoff decision algorithms, namely: SAW (Simple Additive Weighting) and GRA (Grey Relational Analysis).
\end{abstract}

\section{INTRODUCTION}

The architecture for the Beyond 3rd Generation (B3G) wireless networks aims to integrate various heterogeneous wireless access networks over an IP (Internet Protocol) backbone. Currently, there are various standardization bodies working towards this vision. Examples include the 3GPP (3rd Generation Partnership Project) [1], 3GPP2 [2], and the IEEE 802.21 [3]. In order to provide seamless mobility, one of the main design issues is the vertical handoff support. Vertical handoff occurs when connections switch from one network to another (e.g., from WLAN to CDMA 1xRTT).

The vertical handoff process involves three main phases [4], [5], namely system discovery, vertical handoff decision, and vertical handoff execution. During the system discovery phase, the mobile terminal determines which networks can be used. These networks may also advertise the supported data rates and Quality of Service (QoS) parameters. Since the users are mobile, this phase may be invoked periodically.

In the vertical handoff decision phase, the mobile terminal determines whether the connections should continue using the existing selected network or be switched to another network. The decision may depend on various parameters including the type of the application (e.g., conversational, streaming), minimum bandwidth and delay required by the application, access cost, transmit power, and the user's preferences.

During the vertical handoff execution phase, the connections in the mobile terminal are re-routed from the existing network to the new network in a seamless manner. This phase also includes the authentication, authorization, and transfer of a user's context information.
Various vertical handoff decision algorithms have been proposed recently. In [6], the vertical handoff decision is formulated as a fuzzy multiple attribute decision making problem. Two ranking methods are proposed: Simple Additive Weighting (SAW) and Technique for Order Preference by Similarity to Ideal Solution (TOPSIS). In [7], the network selection for vertical handoff is modeled by the Analytic Hierarchy Process (AHP) and the Grey Relational Analysis (GRA). In [8], a performance comparison among SAW, TOPSIS, GRA, and the Multiplicative Exponent Weighting (MEW) for vertical handoff decision is presented. In [4], the handoff decision mechanism is formulated as an optimization problem. Each candidate network is associated with a cost function which depends on a number of criteria, including the bandwidth, delay, and power requirement. An application oriented vertical handoff decision mechanism is proposed in [5]. A multi-layer framework for vertical handoff is proposed in [9]. In [10], a utility-based strategy for network selection is proposed. In [11], the vertical handoff decision is evaluated via a handoff cost function and a handoff threshold function which can be adapted to changes in the network environment dynamically.

Although there have been various vertical handoff algorithms proposed in the literature, our work is motivated by two particular aspects. First, the connection duration needs to be taken into account during the vertical handoff decision. Second, the processing and signaling load during the vertical handoff execution also needs to be taken into consideration. Our work aims to incorporate these two aspects in the model formulation of vertical handoff.

In this paper, we propose a vertical handoff decision algorithm for heterogeneous wireless networks. The problem is formulated as a Markov decision process. There is a link reward function associated with the QoS of the connection. There is also a signaling cost function associated with the signaling overhead and processing load when vertical handoff is performed. The contributions of this paper are as follows:

1) The proposed model is adaptive and applicable to a wide range of conditions. Different link reward functions can be assigned to various applications (e.g., real-time, non-real-time) and networks (e.g., WLAN, CDMA) with different QoS. Different signaling cost functions can be used based on the complexity of the re-routing operation and the signaling load incurred on the network.

2) Numerical results show good performance improvement 


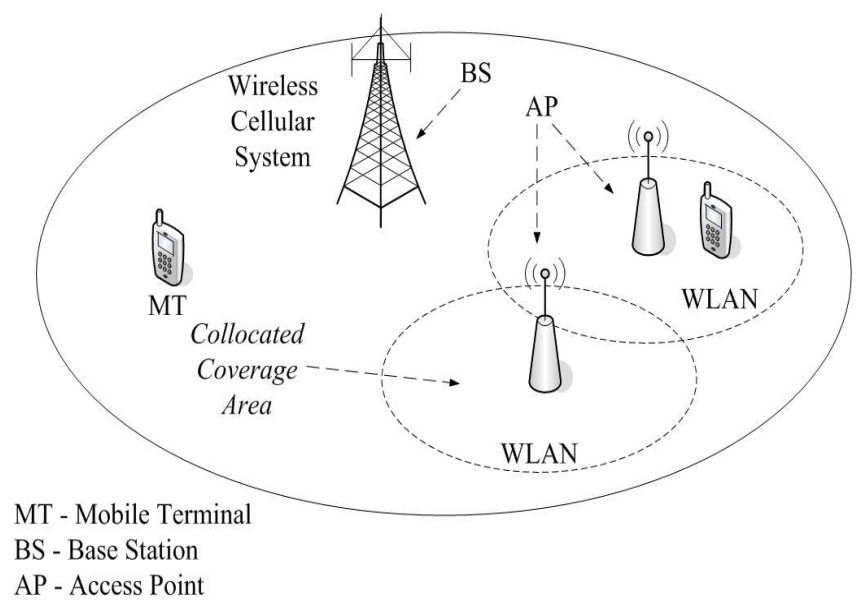

Fig. 1. Heterogeneous wireless networks.

of our proposed scheme over several vertical handoff decision algorithms, namely: SAW (Simple Additive Weighting) [6] and GRA (Grey Relational Analysis) [7].

This paper is organized as follows. The model formulation is presented in Section II. The vertical handoff decision algorithm is described in Section III. Numerical results are presented in Section IV. Conclusions are given in Section V.

\section{Model Formulation}

Each mobile connection may experience a number of vertical handoffs during its connection lifetime. The envisioned heterogeneous wireless environment considered is shown in Fig. 1, where a WLAN is collocated inside the coverage of a wireless cellular system. This specific area is referred to as the collocated coverage area. The mobile terminal is assumed to receive information from the collocated networks within its receiving range periodically. The advertised information from each network may include the available bandwidth and the average delay which can be estimated by the IETF IP performance metrics procedures [12]. Every time period, the mobile terminal decides whether the connection should use the current selected network or be re-routed to another network, which can provide better performance (e.g., lower cost, higher QoS guarantee). The re-routing of the connection from one network to another is a complex process. It increases the processing and signaling load of the network. Thus, there is a tradeoff between the QoS of the connection and the processing and signaling load.

We now describe how to formulate the above vertical handoff decision problem as a Markov decision process (MDP). The notations that we use follow those described in [13]. An MDP model consists of five elements: decision epochs, states, actions, transition probabilities, and rewards. The mobile terminal has to make a decision whenever a certain time period has elapsed. Referring to Fig. 2, the sequence $T=\{1,2, \ldots, N\}$ represents the times of successive decision epochs. The random variable (r.v.) $N$ denotes the time that the connection terminates. At each decision epoch, the mobile terminal has to decide whether the connection should use the current chosen network or be re-routed to another network. Let $M$ denote the total number of collocated networks. The action set $A=\{1,2, \cdots, M\}$. The r.v. $Y_{t}$ denotes the action chosen at decision epoch $t$.

The mobile terminal chooses an action based on its current state information denoted by $S$. For each state $s \in S$, the state information includes the network ID that the device is currently connected to, the bandwidth and delay provided by all the collocated networks. The r.v. $X_{t}$ denotes the state at decision epoch $t$. Given that the current state is $s$ and the chosen action is $a$, the state transition probability function for the next state $s^{\prime}$ is denoted by $P\left[s^{\prime} \mid s, a\right]$. This function is Markovian since it only depends on the current state and action.

The link reward function $f\left(X_{t}, Y_{t}\right)$ reflects the QoS provided by the chosen network to the connection within the time interval from $t$ to $t+1$. The signaling cost function $g\left(X_{t}, Y_{t}\right)$ captures the processing and signaling load incurred when the connection switches from one network to another. If the connection remains using the same network during the interval $(t, t+1)$, then $g\left(X_{t}, Y_{t}\right)$ is equal to zero. For convenience, we define $r\left(X_{t}, Y_{t}\right)=f\left(X_{t}, Y_{t}\right)-g\left(X_{t}, Y_{t}\right)$.

A decision rule prescribes a procedure for action selection in each state at a specified decision epoch. Deterministic Markovian decision rules are functions $\delta_{t}: S \rightarrow A$, which specify the action choice when the system occupies state $s$ at decision epoch $t$. A policy $\pi=\left(\delta_{1}, \delta_{2}, \ldots, \delta_{N}\right)$ is a sequence of decision rules to be used at all decision epochs.

Let $v^{\pi}(s)$ denote the expected total reward between the first decision epoch till the connection termination, given that the policy $\pi$ is used with initial state $s$. We have,

$$
v^{\pi}(s)=E_{s}^{\pi}\left[E_{N}\left\{\sum_{t=1}^{N} r\left(X_{t}, Y_{t}\right)\right\}\right]
$$

where $E_{s}^{\pi}$ denotes the expectation with respect to $\pi$ and initial state $s$, and $E_{N}$ with respect to r.v. $N$.

The r.v. $N$, which denotes the connection termination time, is assumed to be geometrically distributed with mean $1 /(1-$ $\lambda)$. As shown in the Appendix, (1) can be written as:

$$
v^{\pi}(s)=E_{s}^{\pi}\left\{\sum_{t=1}^{\infty} \lambda^{t-1} r\left(X_{t}, Y_{t}\right)\right\}
$$

where $\lambda$ is the discount factor, and $0 \leq \lambda<1$.

Since our optimization problem is to maximize the expected total discounted reward, we define a policy $\pi^{*}$ to be optimal in $\Pi$ if $v^{\pi^{*}}(s) \geq v^{\pi}(s)$ for all $\pi \in \Pi$. A policy is said to be stationary if $\delta_{t}=\delta$ for all $t$. A stationary policy has the form $\pi=(\delta, \delta, \cdots)$; for convenience we denote $\pi$ simply by $\delta$. Our objective is to determine an optimal stationary deterministic policy $\delta^{*}$ which maximizes the expected total discounted reward given by (2). For the rest of the paper, we refer to (2) as the expected total reward. We refer to $\delta^{*}$ as the MDP optimal policy. Note that $\delta^{*}$ is optimal under the expected total discounted reward optimality criterion [13]. 


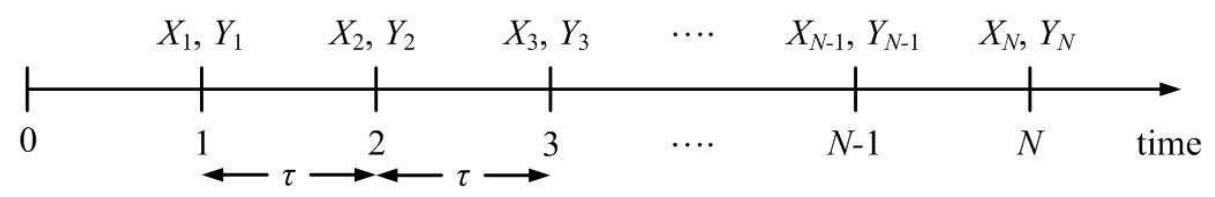

Fig. 2. Timing diagram of a Markov Decision Process (MDP).

\section{Vertical HaNdofF DeCision Algorithm}

\section{A. State, Reward Function, and Transition Probability}

In our proposed vertical handoff decision algorithm, the state space $S$ is defined as:

$S=\{1,2, \cdots, M\} \times B^{1} \times D^{1} \times B^{2} \times D^{2} \cdots \times B^{M} \times D^{M}$

where $M$ denotes the number of available collocated networks, $B^{m}$ and $D^{m}$ denote the set of the available bandwidth and delay from network $m$ (with $m=1,2, \cdots, M$ ), respectively.

To reduce the number elements in $S$, we assume that the bandwidth and delay information is provided in a multiple of units of bandwidth and delay. Specifically, we define:

$$
B^{m}=\left\{1,2,3, \cdots, b_{\max }^{m}\right\}, \quad m=1,2, \cdots, M
$$

and

$$
D^{m}=\left\{1,2,3, \cdots, d_{\max }^{m}\right\}, \quad m=1,2, \cdots, M
$$

where $b_{\max }^{m}$ denotes the maximum available bandwidth provided to a connection by network $m$ and $d_{\max }^{m}$ the maximum delay provided to a connection by network $m$.

Given the current state $s$ and the chosen action $a$, the link reward function $f(s, a)$ is defined as:

$$
f(s, a)=\omega f_{b}(s, a)+(1-\omega) f_{d}(s, a) .
$$

where $\omega$ is the weight factor and $0 \leq \omega \leq 1, f_{b}(s, a)$ denotes the bandwidth function, and $f_{d}(s, a)$ the delay function.

Let $s=\left[i, b_{1}, d_{1}, \cdots, b_{M}, d_{M}\right]$ denote the current state vector. We have

$$
f_{b}(s, a)= \begin{cases}1, & b_{a} \geq U_{B} \\ \left(b_{a}-L_{B}\right) /\left(U_{B}-L_{B}\right), & L_{B}<b_{a}<U_{B} \\ 0, & b_{a} \leq L_{B}\end{cases}
$$

where $L_{B}$ and $U_{B}$ denote the minimum and maximum bandwidth required by the connection, respectively. Similarly,

$$
f_{d}(s, a)=\left\{\begin{array}{lc}
1, & 0<d_{a} \leq L_{D} \\
\left(U_{D}-d_{a}\right) /\left(U_{D}-L_{D}\right), & L_{D}<d_{a}<U_{D} \\
0, & d_{a} \geq U_{D}
\end{array}\right.
$$

where the constants $L_{D}$ and $U_{D}$ denote the minimum and maximum delay required by the connection, respectively.

Let $i$ denote the current network used by the connection. The signaling cost function $g(s, a)$ is defined as:

$$
g(s, a)= \begin{cases}K_{i, a}, & i \neq a \\ 0, & i=a\end{cases}
$$

where $K_{i, a}$ is the switching cost (including re-routing operations and signaling load) from the current network $i$ to the new network $a$.
Between two successive vertical handoff decision epochs, the reward function $r(s, a)$ is defined as:

$$
r(s, a)=f(s, a)-g(s, a) .
$$

Given that the current state $s=\left[i, b_{1}, d_{1}, \cdots, b_{M}, d_{M}\right]$ and the chosen action is $a$, the probability function that the next state $s^{\prime}=\left[j, b_{1}^{\prime}, d_{1}^{\prime}, \cdots, b_{M}^{\prime}, d_{M}^{\prime}\right]$ is given by:

$$
P\left[s^{\prime} \mid s, a\right]= \begin{cases}\prod_{m=1}^{M} P\left[b_{m}^{\prime}, d_{m}^{\prime} \mid b_{m}, d_{m}\right], & j=a \\ 0, & j \neq a\end{cases}
$$

In (8), we assume that the joint bandwidth and delay probability function of each network is independent. This is due to the fact they are managed by different network operators and use different access technologies.

\section{B. Optimality Equations and the Value Iteration Algorithm}

Let $v(s)$ denote the maximum expected total reward given the initial state $s$. That is,

$$
v(s)=\max _{\pi \in \Pi} v^{\pi}(s)
$$

From [13], the optimality equations are given by:

$$
v(s)=\max _{a \in A}\left\{r(s, a)+\sum_{s^{\prime} \in S} \lambda P\left[s^{\prime} \mid s, a\right] v\left(s^{\prime}\right)\right\} .
$$

There are various algorithms available to solve (10). The following value iteration algorithm (VIA) from [13] determines a stationary deterministic optimal policy and the corresponding expected total reward. The solutions of the optimality equations correspond to the maximum expected total reward $v(s)$ and the optimal policy $\delta^{*}(s)$. Note that the MDP optimal policy $\delta^{*}(s)$ indicates the decision to choose which network given the current state is $s$.

\section{Algorithm:}

1) Set $v^{0}(s)=0$ for each state $s$. Specify $\varepsilon>0$ and set $k=0$.

2) For each state $s$, compute $v^{k+1}(s)$ by

$$
v^{k+1}(s)=\max _{a \in A}\left\{r(s, a)+\sum_{s^{\prime} \in S} \lambda P\left[s^{\prime} \mid s, a\right] v^{k}\left(s^{\prime}\right)\right\} .
$$

3) If $\left\|v^{k+1}-v^{k}\right\|<\varepsilon(1-\lambda) /(2 \lambda)$, go to step 4. Otherwise, increment $k$ by 1 and return to step 2 .

4) For each $s \in S$, compute the stationary optimal policy $\delta(s)=\arg \max _{a \in A}\left\{r(s, a)+\sum_{s^{\prime} \in S} \lambda P\left[s^{\prime} \mid s, a\right] v^{k+1}\left(s^{\prime}\right)\right\}$ and stop.

The function norm is defined as $\|v\|=\max |v(s)|$ for $s \in S$. Convergence of the VIA is ensured since the operation 
in Step 2) corresponds to a contraction mapping. Thus, the function $v^{k}(s)$ converges in norm to $v(s)$. Note that the convergence rate of the VIA is linear.

\section{Numerical Results AND Discussions}

The performance of the MDP optimal policy $\delta^{*}$ is compared with SAW [6] and GRA [7]. We denote these policies as $\delta^{S A W}$ and $\delta^{G R A}$, respectively. In addition, two other heuristic policies are included. For the first heuristic, the network to be selected at each decision epoch is the one which has the highest available bandwidth. We denote this policy as $\delta^{B A N}$. For the second heuristic, no vertical handoff is performed during the connection lifetime. We denote this policy as $\delta^{N E V}$.

The performance metrics are the expected total reward per connection and the expected number of vertical handoffs per connection. The expected total reward is defined in Section II. By following the notations in Section III-A, let the state at time $t$ be denoted as $s_{t}=\left[i(t), b_{1}(t), d_{1}(t), \cdots, b_{M}(t), d_{M}(t)\right]$. The expected number of vertical handoffs per connection given policy $\delta$ with initial state $s$ is given by:

$$
\varsigma^{\delta}(s)=E_{s}^{\delta}\left\{\sum_{t=1}^{\infty} \lambda^{t-1} \cdot \mathbf{1}\left[a_{t} \neq i(t)\right]\right\}
$$

where $\mathbf{1}[\cdot]$ is the indicator function (i.e., $\mathbf{1}\left[a_{t} \neq i(t)\right]$ is equal to 1 if $a_{t}$ is not equal to $i(t)$ at time $t$, and is 0 otherwise).

The time between successive decision epochs is assumed to be 15 seconds. There are two collocated networks (i.e., $M=2$ ). Network 1 is a WLAN with $b_{\max }^{1}=25$ and $d_{\max }^{1}=8$. Network 2 is a wireless cellular system with $b_{\max }^{2}=10$ and $d_{\max }^{2}=8$. For simplicity, the switching costs in the signaling cost function (6) are the same (i.e., $K_{1,2}=K_{2,1}$ ). The application is assumed to be voice (i.e., conversational) and $\omega=0.25$ in the link reward function (3). In the bandwidth function (4), $L_{B}=2$ units and $U_{B}=4$ units, where one unit of bandwidth is equal to $16 \mathrm{kbps}$. In the delay function (5), $L_{D}=2$ units and $U_{D}=7$ units, where one unit of delay is equal to $60 \mathrm{~ms}$. Thus, the target delay is less than $150 \mathrm{~ms}$. The connection is still acceptable if the delay is between $150 \mathrm{~ms}$ and $400 \mathrm{~ms}$. The quality of the connection is not acceptable if the delay exceeds 400 $m s$ [14]. Note that the parameters of the link reward function can be modified according to the particular application in use (e.g., voice, video, audio streaming). The initial state at the beginning of the connection is assumed to be $48 \mathrm{kbps}$ of bandwidth and $60 \mathrm{~ms}$ of delay.

For the state transition probability function of the wireless cellular system (i.e., network 2) in (8), we assume that the values of bandwidth and delay are guaranteed for the duration of the connection. Thus,

$$
P\left[b_{2}^{\prime}, d_{2}^{\prime} \mid b_{2}, d_{2}\right]= \begin{cases}1, & b_{2}^{\prime}=b_{2}, d_{2}^{\prime}=d_{2} \\ 0, & \text { otherwise. }\end{cases}
$$

For the state transition probability function of the WLAN, however, we cannot make the same assumption as in the wireless cellular system. Instead, we follow a simulationbased approach to estimate such probabilities. A typical IEEE
802.11 $b$ WLAN is simulated by using the ns-2 (2.29) network simulator [15], where users arrive and depart from the network according to a Poisson process with an average rate of 0.2 users per second. The user's traffic is $64 \mathrm{kbps}$ Constant Bit Rate (CBR). The basic rate and date rate of the WLAN are 1 Mbps and $11 \mathrm{Mbps}$, respectively. The available bandwidth is calculated from the WLAN's capacity, which is approximated to be the achievable throughput by the WLAN under saturation, minus the aggregated traffic of users. The average packet delay is calculated from a mobile terminal to a destination in the wired network. The values of the bandwidth and delay are rounded according to the units defined earlier. The counting of transitions among states is performed to estimate the state transition probabilities.

For the VIA, $\varepsilon$ is chosen to be equal to $10^{-3}$. From the MDP optimal policy, the VIA is used again to determine the expected number of vertical handoffs by solving (11). For the other two vertical handoff decision algorithms SAW and GRA, the ranking of each network is used to determine the policies $\delta^{S A W}$ and $\delta^{G R A}$. Given the reward functions and the state transition probabilities, the corresponding expected total reward and the expected number of vertical handoffs can be determined from (2) and (11), respectively. Similar steps are used to determine $\delta^{B A N}$ and $\delta^{N E V}$.

\section{A. Results}

Fig. 3 and 4 show the expected total reward and the expected number of vertical handoffs versus the switching cost $K_{i, a}$, respectively. The average connection duration is $10 \mathrm{~min}$ (i.e., $\lambda=0.975$ ). Fig. 3 shows that when $K_{i, a}$ increases, the policy $\delta^{*}$ obtained from MDP algorithm gives the highest expected total reward per connection compared to the other algorithms. Fig. 4 shows that when $K_{i, a}$ increases, there is less incentive to perform vertical handoff and the MDP algorithm chooses not to switch more often. On the other hand, $\delta^{S A W}$ and $\delta^{G R A}$ select the network based on the current available bandwidth and delay, and do not take the switching cost into consideration. $\delta^{B A N}$ only considers the available bandwidth and $\delta^{N E V}$ never performs a vertical handoff. Thus, the expected number of vertical handoffs is constant for those algorithms. Recall that an increase in the number of vertical handoffs is directly related to an increase in the signaling load incurred in the networks.

The switching costs $K_{i, a}$ in the signaling cost function (6) provide flexibility for network operators. The values of $K_{i, a}$ can be selected to reflect the complexity of the re-routing operations and the signaling load incurred on the network when vertical handoffs are performed. Small values of $K_{i, a}$ can be set among networks with roaming or interworking agreements. Large values of $K_{i, a}$ can be set temporarily for overloaded networks to deter roaming users from connecting to them.

Fig. 5 and 6 show the expected total reward and the expected number of vertical handoffs versus the discount factor $\lambda$, respectively. Recall that in the discrete-time MDP model, we assume that the time unit (i.e., the time between successive 


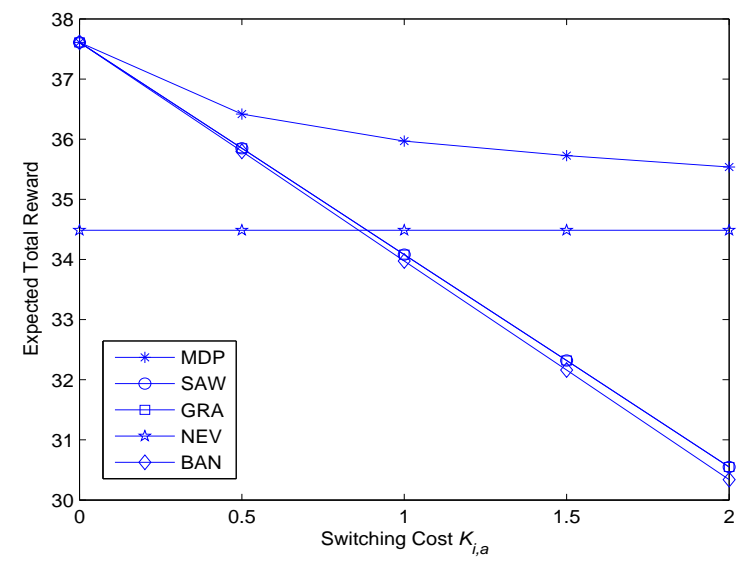

Fig. 3. Expected total reward versus switching cost $K_{i, a} \cdot(\lambda=0.975$ and $\omega=0.25$.)

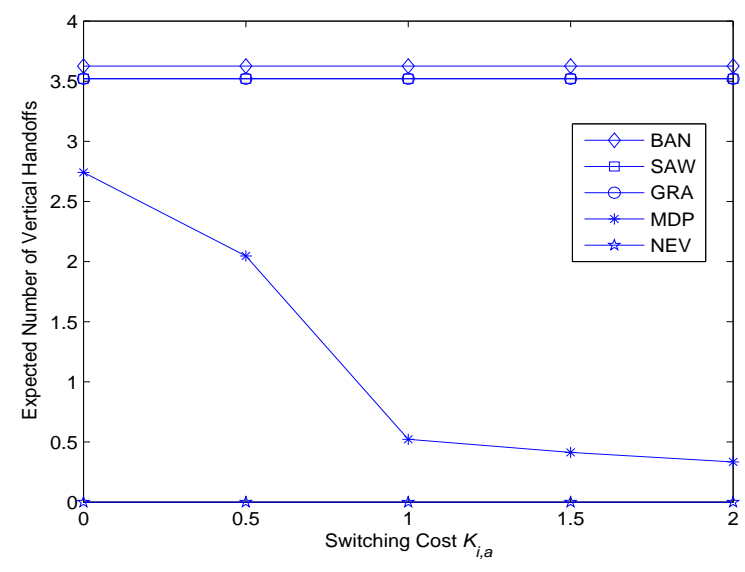

Fig. 4. Expected number of vertical handoffs versus switching cost $K_{i, a}$. $(\lambda=0.975$ and $\omega=0.25$. $)$

decision epochs) is $1 / 4 \mathrm{~min}$. The average connection duration is equal to $1 /(1-\lambda)$ time unit. When $\lambda$ is varied from 0.9 to 0.983 , it corresponds to the variation of the average connection duration from $2.5 \mathrm{~min}$ to $15 \mathrm{~min}$. Fig. 5 shows that the policy $\delta^{*}$ gives the highest expected total reward per connection for all values of $\lambda$. As an example, when the average connection duration is $15 \mathrm{~min}$, the MDP algorithm gives $4.4 \%$ more total expected reward than policies $\delta^{S A W}$ and $\delta^{G R A}, 4.7 \%$ more than $\delta^{B A N}$ and $5 \%$ more than $\delta^{N E V}$. Finally, when the average connection duration increases, the number of decision epochs and the difference in the total expected reward also increase. Thus, the expected number of vertical handoffs increases for all algorithms.

Fig. 7 shows the expected total reward versus the weight factor $\omega$. The policy $\delta^{*}$ from MDP gives the highest expected total reward per connection for all different values of $\omega$. From equation (3), when $\omega$ increases, the bandwidth reward function (4) is more important than the delay reward function (5). As we can see, when $\omega \geq 0.25$, the total expected reward and the expected number of vertical handoffs of $\delta^{S A W}$ and $\delta^{S A W}$ converge to $\delta^{B A N}$. This is because more than $25 \%$ of the importance is placed on the available bandwidth.

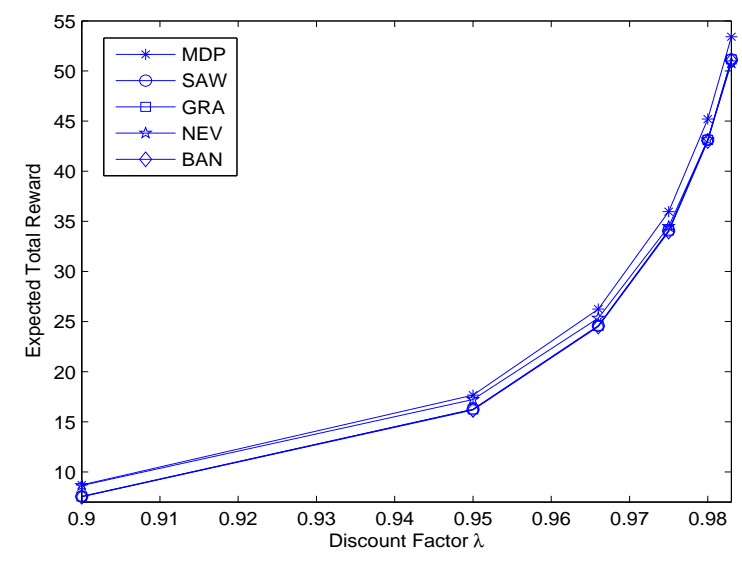

Fig. 5. Expected total reward versus discount factor $\lambda$. $\left(K_{1,2}=1, \omega=\right.$ 0.25$.

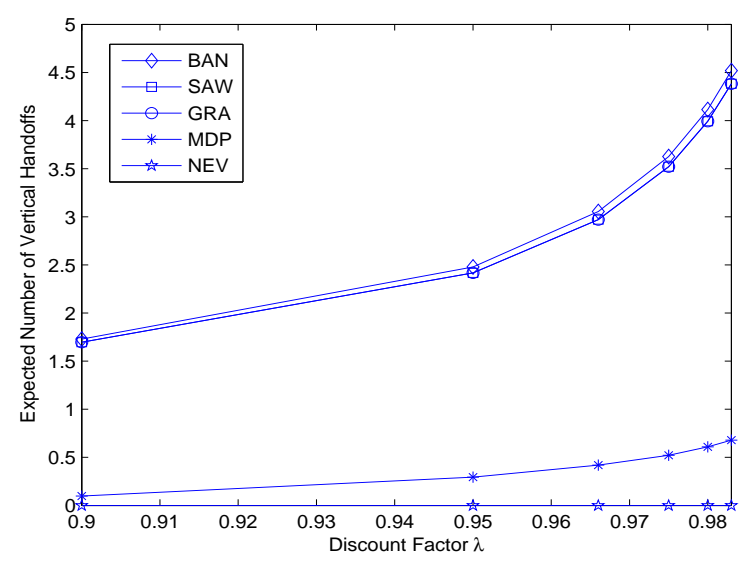

Fig. 6. Expected number of vertical handoffs versus discount factor $\lambda$. $\left(K_{1,2}=1, \omega=0.25\right.$.)

\section{B. Sensitivity Analysis}

In order to calculate the maximum expected total reward of a connection, the MDP optimal policy $\delta^{*}$ needs to be determined. The optimal policy depends on different parameters. Although the parameters such as $K_{i, a}$ can be determined by the network and the parameters such as $\omega$, $L_{B}, L_{D}, U_{B}, U_{D}$ can be assigned by the user or set beforehand by the application, the value of $\lambda$ may not always be estimated correctly by the mobile terminal during connection setup. In that case, the optimal policy may not indeed be the optimal one. In this section, we determine the percentage change of the expected total reward to the variation of the average connection duration. The procedures are as follows:

1) Given the average connection duration $c=(1-\lambda)^{-1}$ and other parameters, we first determine the maximum expected total reward, denoted as Reward (optimal).

2) Let $\hat{c}$ denote the estimated average connection duration and $\Delta_{c}$ denote the percentage change of the average connection duration. These parameters are related by $\hat{c}=\left(1+\Delta_{c}\right) c$. Based on the estimated average connection duration $\hat{c}$ and other parameters, the suboptimal 


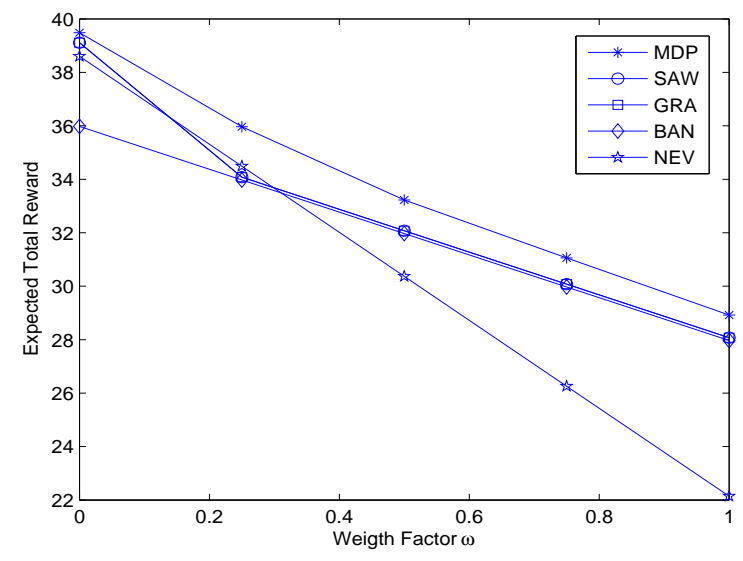

Fig. 7. Expected total reward versus weight factor $\omega .\left(K_{1,2}=1, \lambda=\right.$ 0.975.)

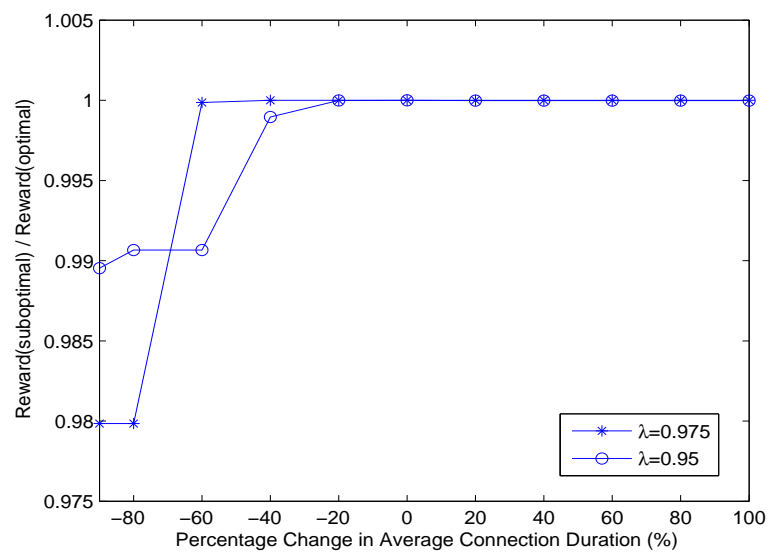

Fig. 8. Variation of the average call duration. $\left(K_{1,2}=1\right.$ and $\omega=0.25$.)

policy is determined. The suboptimal expected total reward, denoted as Reward (suboptimal), is calculated.

3) The change in the expected total reward with respect to the variation of the average connection duration is characterized by the reward ratio, which is defined as Reward (suboptimal) / Reward (optimal).

The results for different $\lambda$ are shown in Fig. 8. Within the $(-95,-40)$ percentage range, there is a small decrease in reward ratio. These results imply that if there is uncertainty in the estimation of the average connection duration, it may be better to over-estimate the connection duration in order to maintain the reward ratio to be close to one.

\section{CONCLUSIONS}

In this paper, we proposed a vertical handoff decision algorithm for heterogeneous wireless networks. Our work incorporated the connection duration and the signaling load incurred on the network for vertical handoff decision. The algorithm is based on the Markov decision process (MDP) formulation with the objective of maximizing the expected total reward of a connection. A stationary deterministic policy is obtained when the connection termination time is geomet- rically distributed. Numerical results show that our proposed MDP algorithm gives a higher expected total reward and lower expected number of vertical handoffs than SAW, GRA, and two heuristic policies under a wide range of conditions.

\section{ACKNOWLEDGMENT}

This work was supported by Bell Canada, the Natural Sciences and Engineering Research Council (NSERC) of Canada, and the Programa de Mejoramiento del Profesorado (PROMEP) from Mexico.

\section{APPENDIX}

\section{A. Expected Total Reward}

In this appendix, we derive the expression $v^{\pi}(s)$, the expected total reward of the connection given a policy $\pi$ and initial state $s$. Assume the r.v. $N$ follows a geometric distribution with mean $1 /(1-\lambda)$. That is, $P(N=n)=$ $\lambda^{n-1}(1-\lambda)$. In this case, (1) can be written as:

$$
v^{\pi}(s)=E_{s}^{\pi}\left\{\sum_{n=1}^{\infty} \sum_{t=1}^{n} r\left(X_{t}, Y_{t}\right) \lambda^{n-1}(1-\lambda)\right\}
$$

Since $\sum_{n=1}^{\infty} \sum_{t=1}^{n}=\sum_{t=1}^{\infty} \sum_{n=t}^{\infty}$, by interchanging the order of the summation, we have

$$
\begin{aligned}
v^{\pi}(s) & =E_{s}^{\pi}\left\{\sum_{t=1}^{\infty} \sum_{n=t}^{\infty} r\left(X_{t}, Y_{t}\right) \lambda^{n-1}(1-\lambda)\right\} \\
& =E_{s}^{\pi}\left\{\sum_{t=1}^{\infty} \lambda^{t-1} r\left(X_{t}, Y_{t}\right)\right\} .
\end{aligned}
$$

\section{REFERENCES}

[1] 3rd Generation Partnership Project (3GPP), http://www.3gpp.org/

[2] 3rd Generation Partnership Project 2 (3GPP2), http://www.3gpp2.org/.

[3] IEEE 802.21 Media Independent Handover Working Group, http://www.ieee802.org/21/.

[4] J. McNair and F. Zhu, "Vertical Handoffs in Fourth-generation Multinetwork Environments," IEEE Wireless Communications, vol. 11, no. 3, pp. 8-15, June 2004.

[5] W. Chen and Y. Shu, "Active Application Oriented Vertical Handoff in Next Generation Wireless Networks," in Proc. of IEEE WCNC'05, New Orleans, LA, March 2005.

[6] W. Zhang, "Handover Decision Using Fuzzy MADM in Heterogeneous Networks," in Proc. of IEEE WCNC'04, Atlanta, GA, March 2004.

[7] Q. Song and A. Jamalipour, "A Network Selection Mechanism for Next Gen. Networks," in Proc. of IEEE ICC'05, Seoul, Korea, May 2005.

[8] E. Stevens-Navarro and V. Wong, "Comparison between Vertical Handoff Decision Algorithms for Heterogeneous Wireless Networks," in Proc. of IEEE VTC'06-Spring, Melbourne, Australia, May 2006.

[9] A. Sur and D. Sicker, "Multi Layer Rules Based Framework for Vertical Handoff," in Proc. of BROADNETS'05, Boston, MA, October 2005.

[10] O. Ormond, J. Murphy, and G. Muntean, "Utility-based Intelligent Network Selection in Beyond 3G Systems," in Proc. of IEEE ICC'06, Istanbul, Turkey, June 2006.

[11] A. Hassawa, N. Nasser, and H. Hassanein, "Tramcar: A Context-Aware Cross-Layer Architecture for Next Generation Heterogeneous Wireless Networks," in Proc. of IEEE ICC'06, Istanbul, Turkey, June 2006.

[12] IETF, "IP Performance Metrics (IPPM) Working Group," http://www.ietf.org/html.charters/ippm-charter.html.

[13] M. Puterman, Markov Decision Processes: Discrete Stochastic Dynamic Programming. John Wiley and Sons, 1994.

[14] ITU-T, "One-way transmision time," Rec. G.114, May 2003

[15] The Network Simulator - ns-2, http://www.isi.edu/nsnam/ns. 